Check for updates

Cite this: RSC Adv., 2018, 8, 31934

\title{
Hyaluronic acid hydrogel scaffolds loaded with cationic niosomes for efficient non-viral gene delivery $\dagger$
}

\author{
Ilia Villate-Beitia, ${ }^{\text {ab }}$ Norman F. Truong, ${ }^{c}$ Idoia Gallego, ${ }^{\text {ad }}$ Jon Zárate, ${ }^{\text {ab }}$ \\ Gustavo Puras, ${ }^{a b}$ José Luis Pedraz $\mathbb{D}^{* a b}$ and Tatiana Segura*ce
}

The lack of ideal non-viral gene carriers has motivated the combination of delivery systems and tissueengineered scaffolds, which may offer relevant advantages such as enhanced stability and reduced toxicity. In this work, we evaluated a new combination between niosome non-viral vectors and hyaluronic acid (HA) hydrogel scaffolds, both widely studied due to their biocompatibility as well as their ability to incorporate a wide variety of molecules. We evaluated three different niosome formulations (niosomes 1, 2 and 3) varying in composition of cationic lipid, helper lipid and non-ionic tensioactives. Niosomes and nioplexes obtained upon the addition of plasmid DNA were characterized in terms of size, polydispersity, zeta potential and ability to transfect mouse bone marrow cloned mesenchymal stem cells (mMSCs) in 2D culture. Niosome 1 was selected for encapsulation in HA hydrogels due to its higher transfection efficiency and the formulation was concentrated in order to be able to incorporate higher amounts of DNA within HA hydrogels. Nioplex-loaded HA hydrogels were characterized in terms of biomechanical properties, particle distribution, nioplex release kinetics and ability to transfect encapsulated mMSCs in 3D culture. Our results showed that nioplex-loaded HA hydrogel scaffolds presented little or no particle aggregation, allowed for extensive cell spreading and were able to efficiently transfect encapsulated mMSCs with high cellular viability. We believe that the knowledge gained through this in vitro model can be utilized to design novel and effective platforms for in vivo local and non-viral gene delivery applications.

Received 14th June 2018 Accepted 31st July 2018

DOI: $10.1039 / c 8 \mathrm{ra05125a}$

rsc.li/rsc-advances fabrication, high reproducibility and acceptable costs, compared to their viral counterparts. ${ }^{4}$ The majority of nanosized non-viral vectors are based on cationic polymers, lipids or peptides. Among the wide variety of non-viral vectors, niosomes have gained interest in recent years due to their high biocompatibility and biodegradability, as well as because of the promising gene transfer results obtained in vivo.,6 Niosomes are synthetic, non-ionic surfactant vesicles with a closed bilayer structure. $^{7}$ They are based on three principal components: (i) cationic lipids, which are responsible for the electrostatic interaction with the negatively charged DNA molecules, ${ }^{8}$ (ii) helper lipids, to improve the physicochemical properties of the suspension $^{9}$ and (iii) non-ionic surfactants, which enhance the stability of the formulation and prevent particle aggregation. ${ }^{10}$ Complexes formed by niosomes and DNA (known as nioplexes) are usually in the range of $100-200 \mathrm{~nm}$ and positively charged, which make them suitable for gene delivery applications. ${ }^{11}$

Complementing gene transfer with matrix design for targeted, local DNA delivery has also gained interest in recent years. Hyaluronic acid (HA), an anionic glycosaminoglycan, is one of the primary components of the natural extracellular matrix (ECM) and it is increasingly gaining popularity as a biomaterial in the field of tissue engineering. ${ }^{12}$ HA hydrogel 
scaffolds have been widely studied for their biocompatibility as well as their ability to incorporate a wide variety of molecules, including nucleic acids. ${ }^{13}$ Non-viral DNA nanoparticles based on cationic polymers such as poly(ethylene imine) (PEI) have been successfully encapsulated into fibrin, ${ }^{\mathbf{1 4 , 1 5}}$ enzymatically degradable PEG hydrogels ${ }^{\mathbf{1 6}}$ and PEG-hyaluronic acid ${ }^{17}$ hydrogels. In addition, cationic nioplexes have also been successfully encapsulated in polysaccharide-based hydrogels made of $\kappa$ carrageenan and of a mixture of methylcellulose and $\kappa$-carrageenan. ${ }^{18}$ However, despite the fast development of niosome formulations in the field of gene delivery, their applicability for encapsulation in HA hydrogel scaffolds has not yet been studied.

Therefore, the main objective of the present work was to develop an effective platform to deliver DNA locally using niosomes as non-viral vectors from HA hydrogel scaffolds. To our knowledge, this is the first example of niosome-based DNA nanoparticle delivery from HA hydrogels for non-viral gene expression. We explored three different niosome formulations varying in composition of cationic lipid, helper lipid and nonionic tensioactives (Fig. 1) in complex with a reporter plasmid encoding for Gaussia luciferase (pGluc) to obtain the nioplexes. The selection of the components, their concentrations and the cationic lipid/DNA mass ratios (w/w) were based on previous studies developed in our laboratory., ${ }^{\mathbf{5}, \mathbf{1 1}}$ Niosomes and corresponding nioplexes were characterized in terms of size, PDI, zeta potential and ability to transfect mouse bone marrow cloned mesenchymal stem cells (mMSCs) in 2D culture. HA hydrogels containing nioplexes were characterized in terms of biomechanical properties, particle distribution and nioplex release kinetics. Additionally, the biological activity of released nioplexes upon hydrogel degradation was also evaluated.

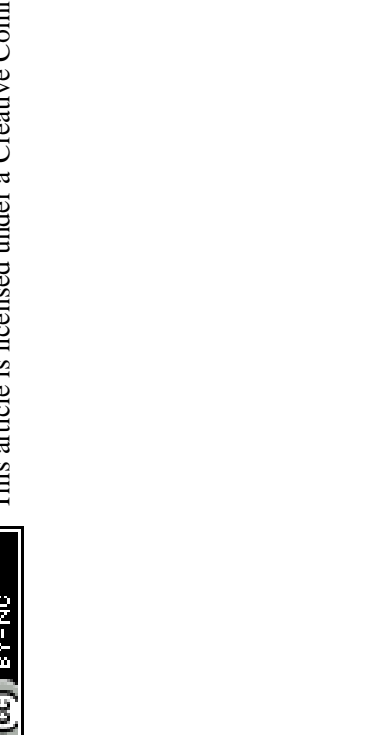

A

Cationic lipids:

mo

NO H Cl

1,2-di-O-octadecenyl-3-trimethylammonium propane

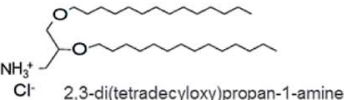

Helper lipids:

$\mathrm{H}_{3} \mathrm{CH}_{3} \mathrm{CH}_{3} \mathrm{CH}_{3} \mathrm{CH}_{3} \mathrm{CH}_{3} \mathrm{CH}_{\mathrm{CH}_{3}}$

Squalene

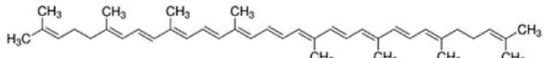

Lycopene

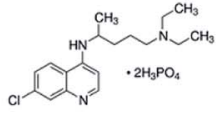

Non-ionic tensioactives:

roh of low to

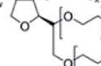

of $\int_{0} \mathrm{CH}_{2}\left(\mathrm{CH}_{2}\right)_{15} \mathrm{CH}_{3}$

$\mathrm{HO}\left(\mathrm{CH}_{2} \mathrm{CH}_{2} \mathrm{O}\right)_{w}, \quad\left(\mathrm{OCH}_{2} \mathrm{CH}_{2}\right)_{\times} \mathrm{OH}$
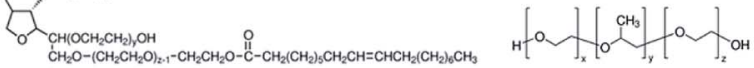

Polysorbate 60

Polysorbate 80

Poloxamer 407

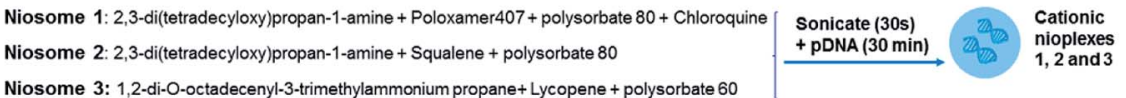

Niosome 3: 1,2-di-O-octadecenyl-3-trimethylammonium propane+Lycopene + polysorbate 60

B

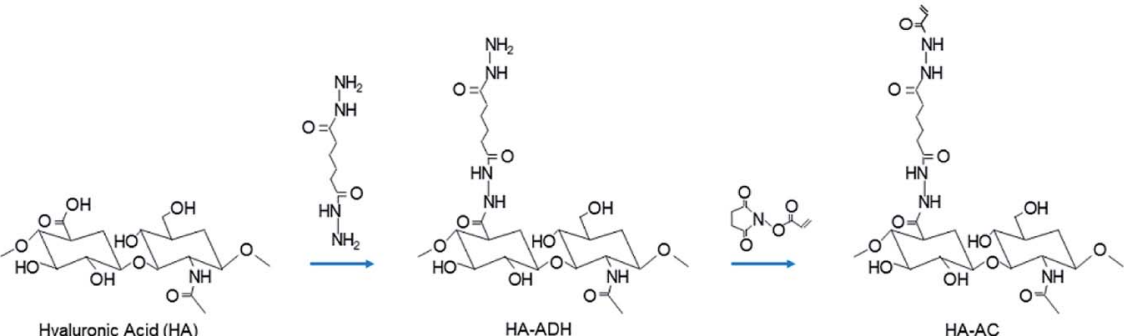

C
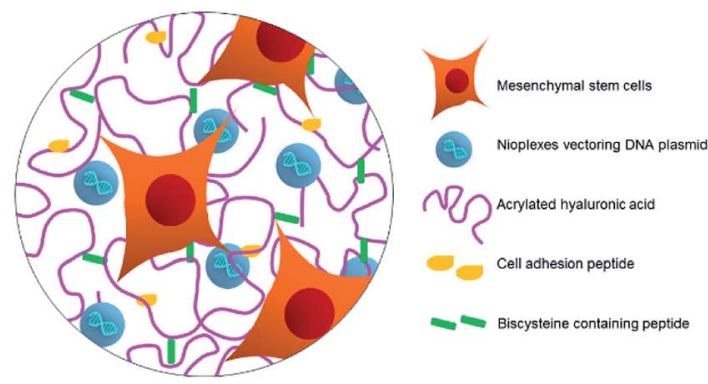

Fig. 1 General scheme of cationic niosomes and hydrogel scaffolds used in this work. (A) Cationic lipid, helper lipid and non-ionic tensioactives components used to elaborate niosome formulations. (B) Modification of HA to obtain HA hydrogels. (C) Representative scheme of a HA hydrogel with entrapped nioplexes. 
mMSCs were efficiently transfected in nioplex encapsulated HA hydrogels and presented excellent cellular viability. These results demonstrate the potential for nioplex loaded HA hydrogels for sustained gene delivery.

\section{Experimental section}

\section{Formation of niosomes and nioplexes}

Formulations based on niosomes 1, 2 and 3 (Fig. 1) were prepared by the oil-in-water emulsion technique as previously described. ${ }^{11}$ The organic phase of niosome 1 contained $5 \mathrm{mg}$ of cationic lipid 2,3-di(tetradecyloxy)propan-1-amine and $12.5 \mathrm{mg}$ of poloxamer 407 (Sigma-Aldrich, USA) and $12.5 \mathrm{mg}$ of polysorbate 80 (Sigma-Aldrich, USA) as non-ionic tensioactives, all dissolved in $1 \mathrm{ml}$ of dichloromethane (DCM) (Panreac, USA). The water phase of niosome 1 contained $2.5 \mathrm{mg}$ of helper lipid chloroquine diphosphate salt (Sigma-Aldrich, USA) dissolved in $5 \mathrm{ml}$ of distilled water. In niosome 2 , the organic phase contained $5 \mathrm{mg}$ of cationic lipid 2,3-di(tetradecyloxy)propan-1amine and $20 \mu \mathrm{l}$ of helper lipid squalene (Sigma-Aldrich, USA), dissolved in $1 \mathrm{ml}$ of DCM; the aqueous phase contained $25 \mathrm{mg}$ of polysorbate 80 dissolved in $5 \mathrm{ml}$ of distilled water. The organic phase of niosome 3 contained $5 \mathrm{mg}$ of cationic lipid 1,2di-O-octadecenyl-3-trimethylammonium propane (DOTMA) (Avanti Polar Lipids, Inc., Alabama, USA), $1.1 \mathrm{mg}$ of helper lipid lycopene (Sigma-Aldrich, USA) and $26 \mathrm{mg}$ of non-ionic tensioactives polysorbate 60 (Sigma-Aldrich, USA), dissolved in $1 \mathrm{ml}$ of DCM, while the water phase consisted of $5 \mathrm{ml}$ of distilled water. The organic phase and the water phase were emulsified by sonication (Branson Sonifier 250, Danbury) for $30 \mathrm{~s}$ at $50 \mathrm{~W}$. The organic solvent was removed from the emulsion by evaporation under magnetic agitation for $3 \mathrm{~h}$ at room temperature, obtaining niosome solutions $\mathbf{1}, \mathbf{2}$ and $\mathbf{3}$ at a concentration of $1 \mathrm{mg}$ of cationic lipid per $\mathrm{ml}$. Niosomes were concentrated using absorbent gel (Spectrum Labs, USA) and Slide-A-Lyzer MINI Dialysis Units of 10000 MWCO (Thermo Scientific, USA). Briefly, $500 \mu \mathrm{l}$ of niosome formulation were introduced in the dialysis units and those were kept in the absorbent gel overnight at $4{ }^{\circ} \mathrm{C}$. Next, the final volume after dialysis was measured.

The nioplexes were formed by mixing an appropriate volume of a stock solution of a Gaussia luciferase expression vector (pGluc) (New England BioLabs, Ipswich, MA) with different volumes of niosomes 1,2 and 3 to obtain, respectively, the following cationic lipid/DNA (w/w) mass ratios: 2/1, 15/1 and 18/ 1. Niosomes and DNA were incubated for $30 \mathrm{~min}$ at room temperature to enhance electrostatic interactions and allow the formation of nioplexes. The pGluc plasmid was expanded using an endotoxin-free Giga Prep kit from Qiagen following the manufacturer's instructions.

\section{Size, polydispersity index, zeta potential and morphology of niosomes and nioplexes}

The intensity mean diameter ( $Z$-average) and the zeta potential of niosomes and nioplexes were determined by dynamic light scattering and by laser Doppler velocimetry, respectively, using a Zetasizer Nano ZS (Malvern Instrument, UK). Briefly, $50 \mu \mathrm{l}$ of the formulations were resuspended into $950 \mu \mathrm{l}$ of $0.1 \mathrm{mM} \mathrm{NaCl}$ solution. All measurements were carried out in triplicate. Particle hydrodynamic diameter was obtained by cumulative analysis. The Smoluchowski approximation was used to support the calculation of the zeta potential from the electrophoretic mobility. The morphology of niosomes was determined by transmission electron microscopy (TEM) as previously described. ${ }^{5}$

\section{Cell culture and 2D transfection}

Mouse bone marrow cloned mesenchymal stem cells (mMSCs, D1, CRL12424) were purchased from ATCC (Manassas, VA) and cultured in Dulbecco's Modified Eagle Medium (DMEM, Invitrogen) supplemented with $10 \%$ bovine growth serum (BGS, Hyclone, Logan, UT) and $1 \%$ penicillin/streptomycin (Invitrogen, Grand Island, NY) at $37{ }^{\circ} \mathrm{C}$ and $5 \% \mathrm{CO}_{2}$. The cells were split using trypsin following standard protocols.

For transfection in 2D cell culture, cells were seeded in 48 well plates at a density of 25000 cells per $\mathrm{ml}$ in a total volume of $250 \mu \mathrm{l}$ per well, and incubated overnight to achieve $70 \%$ of confluence at the time of transfection with nioplexes. Nioplexes were suspended in OptiMEM (Gibco, San Diego, CA, USA) transfection medium. Then, growth medium was removed from the plate and cells were exposed for $4 \mathrm{~h}$ to nioplexes at $37^{\circ} \mathrm{C}$ and $5 \% \mathrm{CO}_{2}$. After the incubation, nioplexes were removed and fresh growth medium was added to the cells. Lipofectamine $2000^{\mathrm{TM}}$ (Fisher Scientific, USA) at cationic lipid/DNA mass ratio of 2/1 was used as a positive control of the transfection process. Non-treated cells were used as a negative control. Transfection efficiency was measured $48 \mathrm{~h}$ after exposure to nioplexes using the Gaussia Luciferase Assay Kit (New England BioLabs, Ipswich, MA) following manufacturer's protocol. Briefly, a $20 \mu \mathrm{l}$ sample was mixed with a $50 \mu \mathrm{l} 1 \times$ substrate solution, pipetted for 2-3 s, and read for luminescence with a $5 \mathrm{~s}$ integration. Background was determined with media that did not contain any DNA, and values were expressed as relative light units (RLU).

\section{HA-acrylate synthesis and formation of HA hydrogels loaded with nioplexes}

Acrylated hyaluronic acid (HA-AC) was prepared using a twostep synthesis as previously described. ${ }^{19}$ The extent of acrylation was determined to be $15 \%$ (ESI Fig. $1 \dagger$ ). HA hydrogels were formed by Michael-type addition of biscysteine-containing AcGCRDGPQGIWGQDRCG-NH2 peptides (MMP) (Genescript, Piscataway, NJ) onto HA-AC functionalized with cell adhesion Ac-GCGWGRGDSPG-NH2 peptides (RGD) (Genescript). A lyophilized aliquot of RGD peptides $(0.1 \mathrm{mg})$ was dissolved in 15 $\mu \mathrm{l}$ of $0.3 \mathrm{M}$ HEPES buffer $(\mathrm{pH}=8.7)$, mixed with HA-AC, and allowed to react for $20 \mathrm{~min}$ at room temperature. The HA-RGD solution was kept in ice until used. A lyophilized aliquot of the cross-linker $(0.91 \mathrm{mg})$ was then diluted in $18.2 \mu \mathrm{l}$ of $0.3 \mathrm{M}$ HEPES buffer $(\mathrm{pH}=8.2)$ immediately before mixing with nioplexes, HA-RGD (final concentration of $500 \mu \mathrm{M}$ RGD), and the cell solution (250 000 cells per $100 \mu \mathrm{l}$ of final gel volume). Three 
different volumes of nioplexes were loaded into the hydrogels, obtaining final DNA concentrations of $0.055 \mu \mathrm{g} \mu \mathrm{l}^{-1}, 0.12 \mu \mathrm{g}$ $\mu \mathrm{l}^{-1}$ and $0.2 \mu \mathrm{g} \mu \mathrm{l}^{-1}$ in hydrogels 2,3 and 4 , respectively. Hydrogel 1 did not contain nioplexes and was used as a control. Hydrogel 3 did not contain HEPES buffer since the whole volume was replaced by nioplexes. Hydrogel 4 did not contain HEPES and neither cell growth medium since both volumes were replaced by nioplexes.

For rheology and particle distribution assays, gels were formed in the absence of cells. Gelation was achieved by placing a $40 \mu \mathrm{l}$ drop of the precursor solution between Sigmacoted glass slides (Sigma-Aldrich, USA) for $30 \mathrm{~min}$ at $37^{\circ} \mathrm{C}$. The final gel was placed inside 48 -well plates for culture. Thorough mixing was used to ensure the nioplexes were uniformly distributed throughout the hydrogel. The gel was then allowed to swell in phosphate-buffered saline (PBS) for 2 hours.

YOYO-1 (Invitrogen, USA), a nucleic acid dye, was used to stain the gels to visualize the distribution of the nioplexes inside the HA hydrogels. The images were taken using the fluorescent (Observer Z1 Zeiss) microscope with $10 \times$ magnification.

\section{Characterization of HA hydrogel mechanical properties}

The storage and loss moduli were measured with a plate-toplate rheometer (Physica MCR, Anton Paar, Ashland, VA) using an $8 \mathrm{~mm}$ plate under a constant strain of 0.01 and frequency ranging from 0.1 to $10 \mathrm{rad} \mathrm{s}^{-1}$. Hydrogels were made as detailed above and cut to $8.0 \mathrm{~mm}$ in diameter to fit the plate. A humid hood was used to prevent the hydrogel from drying, and the temperature was maintained at $37^{\circ} \mathrm{C}$ throughout the measurement.

\section{Radiolabeling DNA}

Plasmid DNA was radiolabeled with dCTP $(100 \mu \mathrm{Ci}$, MP Biomedicals, Santa Ana, CA) using a Nick translation kit (Roche, Indianapolis, IN) as per the manufacturer's protocol. Briefly, an equimolar mixture of dATP, dGTP, dTTP, and ${ }^{32} \mathrm{P}$-dCTP was prepared and added to the DNA $(1 \mu \mathrm{g})$ solution. Once the enzyme solution was added to the mixture, the final solution $(200 \mu \mathrm{l})$ was gently mixed by pipetting and incubated for $2 \mathrm{~h}$ at $15{ }^{\circ} \mathrm{C}$. The reaction was stopped by addition of $10 \mu \mathrm{l}$ of $0.2 \mathrm{M}$ ethylenediaminetetraacetic acid (EDTA, $\mathrm{pH}=8.0$ ) and heating to $65{ }^{\circ} \mathrm{C}$ for $10 \mathrm{~min}$. The DNA was purified using the DNA concentrator kit (Zymo Research, Irvine, CA) following the manufacturer's instructions. The radiolabeled ("hot") DNA concentration was $0.04 \mu \mathrm{g} \mu^{-1}$. This "hot" DNA was then mixed with non-radiolabeled DNA to a concentration of $0.25 \%$ "hot" DNA.

\section{Nioplex release kinetics and activity}

To determine the extent of release of the encapsulated nioplexes and their activity post encapsulation, gels were formed as described above with $1 \%$ radiolabeled DNA. The gels were swelled in PBS for $2 \mathrm{~h}$ and the swelling solution was collected. The gels were then placed in $200 \mu \mathrm{l}$ of release solution, either PBS only or $1 \mathrm{U} \mathrm{ml}^{-1}$ collagenase I in PBS. At the indicated time points, $200 \mu \mathrm{l}$ of the solution was removed, and replaced with fresh release solution. DNA concentrations of collected release solutions and from remaining hydrogels were measured using a scintillation counter. The readout was analyzed using a standard curve.

To determine the activity of the encapsulated nioplexes, a HA gel was prepared and swelled as indicated above using the pGluc plasmid. After swelling in PBS, the gel was degraded through incubation with $100 \mu \mathrm{l}$ of $0.25 \%$ trypsin at $37{ }^{\circ} \mathrm{C}$ for $10 \mathrm{~min}$. The collected nioplexes from the degraded hydrogel sample were then used for a bolus transfection $(0.625 \mu \mathrm{g}$ of DNA per well for a 48 well-plate) and compared to freshly made nioplexes. The cell media was collected after $48 \mathrm{~h}$, and transgene expression was measured using the Gaussia Luciferase Assay Kit (New England BioLabs, Ipswich, MA) as described.

\section{Gene transfer, cell viability and cell spreading in 3D culture}

Control hydrogel 1 without nioplexes and nioplex-loaded hydrogels $\mathbf{2}$ and $\mathbf{3}$ with mMSCs were made as described above, and transfection efficiency was measured at $48 \mathrm{~h}$. Secreted Gluc levels in the media were quantified using a BioLux Gaussia Luciferase Assay Kit (New England BioLabs, Ipswich, MA) as described.

Cell viability in hydrogels 1-3 was studied using a LIVE/ DEAD kit (Molecular Probes, Eugene, OR). Briefly, $2 \mu \mathrm{l}$ of ethidium homodimer-1 and $0.5 \mu$ of calcein AM from the kit were diluted with $1 \mathrm{ml}$ of DMEM. Each gel was stained with 150 $\mu \mathrm{l}$ of this staining solution for $30 \mathrm{~min}$ at $37^{\circ} \mathrm{C}$ in the dark. Cell viability from hydrogels 2 and 3 was normalized to the cell viability from control hydrogel 1 which contained no nioplexes. To better analyze cell spreading, separate gels were fixed for $30 \mathrm{~min}$ at room temperature using $4 \%$ paraformaldehyde, rinsed with PBS, treated with $0.1 \%$ Triton-X for $10 \mathrm{~min}$, and stained for $90 \mathrm{~min}$ in the dark with $4^{\prime}, 6$-diamidino-2phenylindole (DAPI) for cell nuclei $(500 \times$ dilution from $5 \mathrm{mg}$ $\mathrm{ml}^{-1}$ stock) and rhodamine-phalloidin for actin filaments (5 $\mu \mathrm{l}$ per $200 \mu \mathrm{l}$ final stain solution) in $1 \%$ bovine serum albumin solution. The samples were then washed with $0.05 \%$ Tween-20. For both cell viability and cell spreading, a Nikon confocal microscope was used to visualize samples. To better visualize the distribution throughout the hydrogel, $z$-stacks $1213 \mu \mathrm{m}$ thick were taken for each image, deconvoluted to minimize background, and presented either as maximum intensity projections or as an aerial view of a 3D render of the $z$-stack.

\section{Statistical analysis}

To analyze the differences, a multiple comparison KruskalWallis test followed by a Mann-Whitney $U$ test was performed. Normal distribution was determined using a Shapiro-Wilks test and homogeneity of variance by the Levene test. Data were expressed as mean $\pm \mathrm{SD}$, unless stated otherwise. A $p$ value $<$ 0.05 was considered statistically significant. The analysis was performed using the IBM SPSS Statistics 22.Ink statistical package. 


\section{Results and discussion}

Since the emergence of non-viral gene delivery from hydrogel scaffolds, emphasis has been placed on optimizing non-viral vectors for combining gene transfer with matrix design and enhancing transfection efficiency. Yet while high concentrations of non-viral DNA complexes in hydrogels have been demonstrated to improve local gene delivery, ${ }^{20}$ the physical incorporation of DNA complexes into hydrogels is challenging due to some limitations such as aggregation and inactivation of the complexes inside hydrogel scaffolds. ${ }^{21}$ Among the wide variety of non-viral vectors, poly(ethylene imine) (PEI) has been successfully encapsulated in HA hydrogels and effective local transgene expression and ability to induce angiogenesis in vivo have been reported. ${ }^{3}$ Although PEI derivatives present high gene carrying capacity and ability to achieve high transfection efficiencies, their biomedical application is often restricted due to immunogenicity and cytotoxicity issues. ${ }^{22}$ In this regard, niosomes offer several advantages, since they have high compatibility with biological systems and low toxicity because of their non-ionic nature and are biodegradable and nonimmunogenic. ${ }^{23}$

The three different niosome formulations used in this work differed in the composition of cationic lipid, helper lipid and non-ionic tensioactives (Fig. 1). These niosome components have previously demonstrated suitability for gene delivery applications. For instance, niosome formulations containing the non-ionic surfactant polysorbate 80 combined with the helper lipid squalene have shown effective gene delivery. ${ }^{5}$ In addition, it has been recently shown that the helper lipid lycopene, combined with cationic lipid DOTMA and polysorbate 60, enhances retinal transfection ${ }^{24}$ and poloxamer 407 has been widely used in drug delivery applications. ${ }^{25}$ The use of chloroquine has also been reported to enhance gene delivery both in vitro and in vivo. ${ }^{26}$ Chloroquine prevents the acidification of endosomes, fusion of endosomes and lysosomes, and inhibits the lysosomal enzymes. ${ }^{27,28}$ Therefore, the selection of the components, and their concentrations used to prepare the niosome formulations, as well as the cationic lipid/DNA mass ratios $(\mathrm{w} / \mathrm{w})$ to form the corresponding nioplexes were based on available data and previous studies developed in our laboratory.

The physicochemical analysis of formulations used in this work revealed that the mean particle sizes of niosomes 1, 2 and 3 were $118.1 \pm 1.8 \mathrm{~nm}, 136.4 \pm 0.9 \mathrm{~nm}$ and $105.7 \pm 2.3 \mathrm{~nm}$, respectively (Fig. 2A). Niosomes 1 and 2 were relatively monodisperse (PDI $<0.2$ ) while niosome 3 formed more polydisperse nanoparticle preparations (PDI $=0.4$ ). All formulations formed highly positively charged nanoparticles with zeta potential values of $+35.9 \pm 2.2 \mathrm{mV},+48.5 \pm 0.6 \mathrm{mV}$ and $+32.9 \pm 1.7 \mathrm{mV}$, for niosomes 1, 2 and 3, respectively (Fig. 2B), necessary to bind to the negatively charged DNA molecules by electrostatic interactions. ${ }^{29,30}$ In addition, positive zeta potential values enhance cellular uptake. ${ }^{31}$ Compared to non-treated cells, all formulations presented ability to transfect but nioplexes based on niosome 1 showed significantly higher transgene expression than nioplexes based on niosomes 2 and 3 (Fig. 2C). Although
A

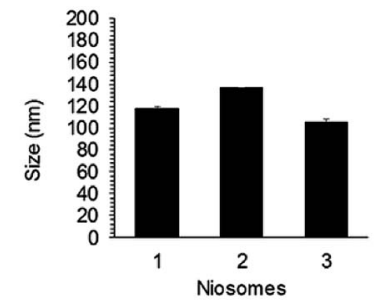

B

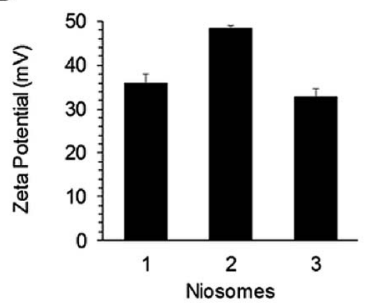

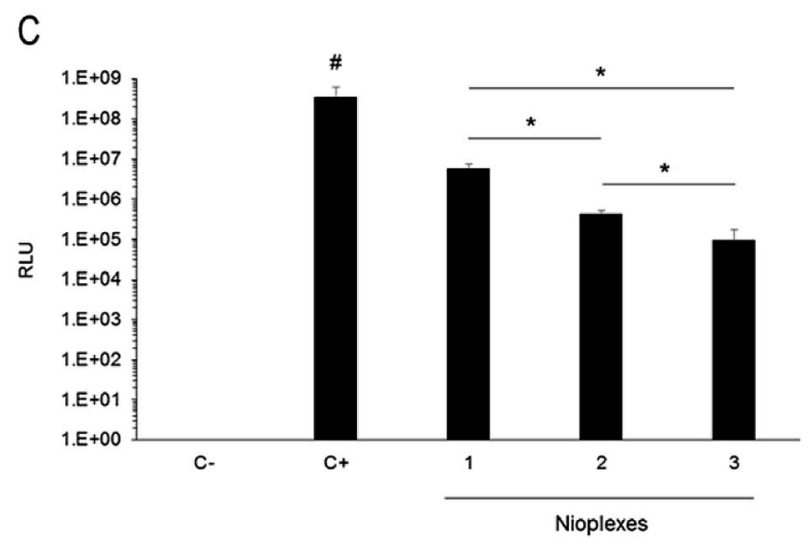

Fig. 2 Screening of niosome formulations. (A) Size. (B) Zeta potential. (C) Transfection efficiency $48 \mathrm{~h}$ post-exposure of mMSCs to nioplexes based on niosomes 1, 2 and 3 at cationic lipid/DNA mass ratios (w/w) 2/1, 15/1 and 18/1, respectively. Positive (Lipofectamine 2000TM at a cationic lipid/DNA mass ratio of $2 / 1$ ) and negative (no DNA) controls are shown for reference. ${ }^{*} p<0.05$ for transfection efficiency between niosome formulations, ${ }^{*} p<0.05$ for transfection efficiency of all niosome formulations relative to the positive control.

transfection levels of niosomes were lower than the positive control Lipofectamine $2000^{\mathrm{TM}}$, the latest is not considered suitable for in vivo gene delivery due to its high cytotoxicity even at low concentrations. ${ }^{32}$ Therefore, we selected the nioplexes based on niosome 1 at 2/1 cationic lipid/DNA mass ratio (w/w) formulation to study its applicability for non-viral gene delivery in HA hydrogels.

The use of nioplexes at low mass ratios presents several advantages, including the possibility of incrementing the dose of DNA as well as decreasing cellular toxicity. ${ }^{33}$ Additionally, in order to be able to incorporate higher, more relevant amounts of DNA within HA hydrogels, the formulation based on niosome 1 was concentrated from $1 \mathrm{mg} \mathrm{ml}^{-1}$ to $2 \mathrm{mg} \mathrm{ml}^{-1}$. The TEM images of the concentrated formulation showed that concentration process did not affect morphology and, as expected, more particles were visible in the concentrated sample compared to the non-concentrated one (Fig. 3A and B). Size and PDI values were also maintained similar in the concentrated formulation. In contrast, zeta potential values declined from $+35.9 \pm 2.2 \mathrm{mV}$ to $+25.77 \pm 1.1 \mathrm{mV}$, but since values remained above $+20 \mathrm{mV}$, the concentrated formulation should not present an increased propensity to form aggregates along the time. ${ }^{34}$ Additionally, the transfection ability of both concentrated and non-concentrated formulations was evaluated at cationic lipid/ DNA mass ratio $2 / 1$ and no statistically significant differences ( $p$ 
A

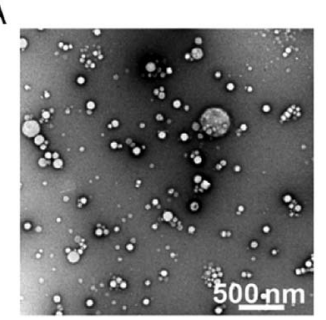

B

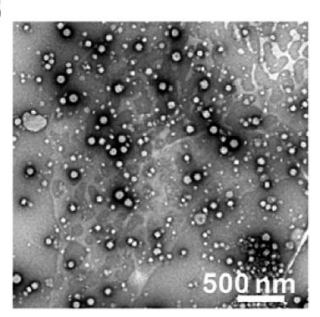

C

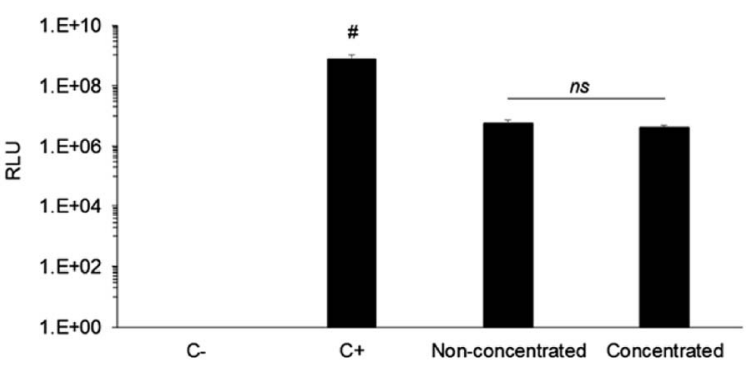

Fig. 3 Characterization of concentrated niosome formulations. (A and B) TEM images of non-concentrated (A) and concentrated (B) formulations based on niosome 1 at $10000 \times$ magnification. Scale bars: $500.0 \mathrm{~nm}$. (C) Transfection efficiency $48 \mathrm{~h}$ post-exposure of mMSCs to nioplexes based on concentrated and non-concentrated niosome 1 at 2/1 cationic lipid/DNA mass ratio (w/w). ${ }^{*} p<0.05$ for transfection efficiency between niosome formulations, ${ }^{\#} p<0.05$ for transfection efficiency of all niosome formulations relative to the positive control.

$>0.05$ ) were found between the transfection efficiencies of both formulations (Fig. 3C), indicating that the concentrating process does not affect to the transfection capacity. In view of these results, we decided to use the concentrated formulation at the low cationic lipid/DNA mass ratio $2 / 1$ for encapsulation in HA hydrogels.

In order to achieve therapeutically relevant levels of DNA, ${ }^{35}$ we evaluated three different amounts of nioplexes based on concentrated niosome 1 at 2/1 cationic lipid/DNA mass ratio, obtaining final DNA concentrations of $0.055 \mu \mathrm{g} \mu \mathrm{l}^{-1}$, $0.12 \mu \mathrm{g} \mu \mathrm{l}^{-1}$ and $0.2 \mu \mathrm{g} \mu \mathrm{l}^{-1}$ in hydrogels 2,3 and 4, respectively. Different DNA concentrations of hydrogels could cause differences in the mechanical properties, which are important factors determining cell proliferation, spreading and transgene expression in hydrogel scaffolds. High hydrogel stiffness (over $800 \mathrm{~Pa}$ ) has been related to reduced cell spreading and transgene expression, while soft hydrogels (200-260 Pa) resulted in enhanced transgene expression. ${ }^{21}$ Therefore, we evaluated the mechanical properties of hydrogels as a function of DNA concentration in order to determine their grade of stiffness. Rheological characterization showed high variability in the mechanical properties of hydrogels ranging from an average modulus of $\sim 380$ Pa to $\sim 1215$ Pa over a frequency range of 0.1$10 \mathrm{rad} \mathrm{s}^{-1}$ at a constant strain of 0.01 (Fig. 4A and B). Hydrogel 3 presented the highest stiffness among the hydrogels tested, while hydrogel $\mathbf{4}$, with the highest DNA concentration, showed to be the softest. Such high values were not expected in hydrogel 3 , however, that condition was not discarded and further
A
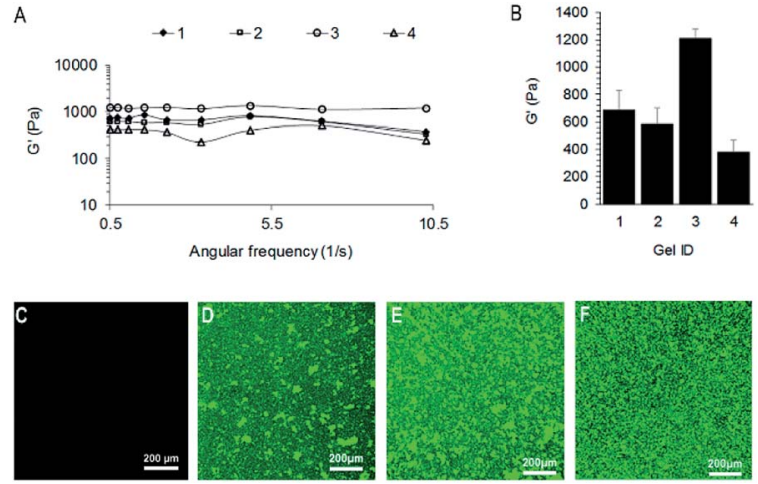

Fig. 4 Characterization of HA hydrogels loaded with nioplexes. (A) The mechanical properties of the hydrogels were determined using plate-to-plate rheometry over a frequency range of $0.1-10 \mathrm{rad} \mathrm{s}^{-1}$ at a constant strain of 0.01. (B) Average storage modulus. (C-F) Particle distribution in hydrogels 1 (C), 2 (D), 3 (E) and 4 (F). Gel ID: 1, control hydrogel without nioplexes; $2,0.055 \mu \mathrm{g}$ DNA per $\mu \mathrm{l} ; 3,0.12 \mu \mathrm{g}$ DNA per $\mu \mathrm{l}$ and $4,0.2 \mu \mathrm{g}$ DNA per $\mu \mathrm{l}$. Scale bars: $200 \mu \mathrm{m}$.

studies were carried out in order to determine its transfection capacity despite its high stiffness. The evaluation of the distribution of the nioplexes inside the hydrogel scaffold (Fig. 4C-F) showed that nioplexes were present uniformly throughout the hydrogels and, in hydrogels 3 and $\mathbf{4}$, those were observed mostly as unaggregated particles (Fig. 4E and F). However, hydrogel 4 did not contain any cell growth medium because it was completely replaced by DNA in order to obtain higher amounts of genetic material. Therefore, it was not a possible candidate for 3D cell culture nor for in vitro transfection assays and only hydrogels 2 and 3 were evaluated for gene delivery into encapsulated mMSCs. Yet, the high DNA concentration and absence of particle aggregation in hydrogel 4 suggested that it could be an attractive option for in vivo gene delivery. Taken together, these results demonstrate that nioplexes can be successfully encapsulated into HA hydrogels without significant particle aggregation, although high variability in mechanical properties was observed.

In order to validate that nioplexes maintained the ability to transfect cells after encapsulation in HA hydrogel 3, we synthesized nioplex-loaded hydrogels, degraded them with trypsin and performed a transfection with the released nioplexes (Fig. 5). Not unexpectedly, nioplexes released from hydrogels degraded with trypsin were still able to efficiently transfect mMSCs in 2D culture, albeit to a somewhat lesser extent than fresh nioplexes (Fig. 5B). When exposing cells to fresh nioplexes + hydrogel + trypsin (Fig. 5C), transfection was similar to that obtained with nioplexes released from degraded hydrogels (Fig. 5B). When exposing cells to fresh nioplexes + trypsin (Fig. 5D), the difference in transfection was less evident. Cells exposed to naked DNA + hydrogel + trypsin (Fig. 5E) and cells exposed to hydrogels without nioplexes + trypsin (Fig. 5F) did not show luminescence signal. Statistical differences were found between all conditions $(p<0.05)$ except for between conditions " $\mathrm{B}$ " and " $\mathrm{C}$ " $(p>0.05)$. These results showed that the transfection capacity of the nioplexes was negatively affected by 


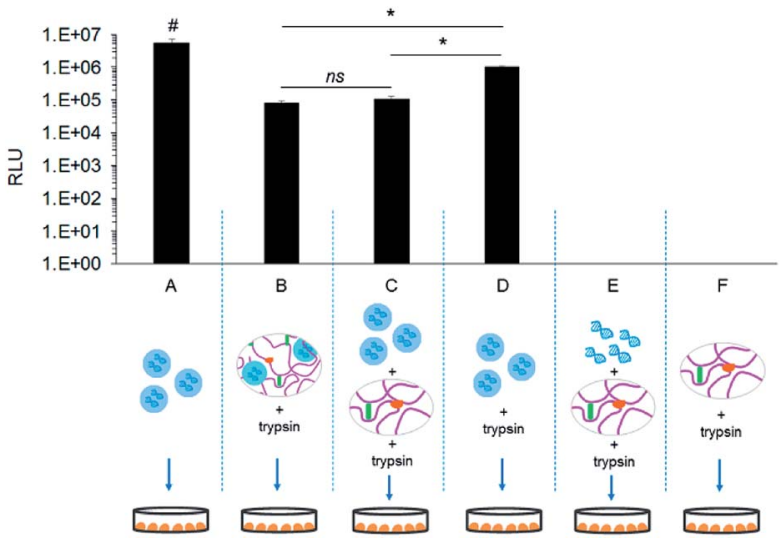

Fig. 5 Biological activity of nioplexes based on concentrated niosome 1 at 2/1 cationic lipid/DNA mass ratio (w/w). (A) Fresh nioplexes. (B) Nioplexes released from degraded HA hydrogel 3 with trypsin. (C) Fresh nioplexes supplemented with degraded hydrogel 3 with trypsin. (D) Fresh nioplexes supplemented with trypsin. (E) Naked DNA supplemented with degraded hydrogel 3 with trypsin. (F) Hydrogel 3 without nioplexes degraded with trypsin. ${ }^{*} p<0.05$ for transfection efficiency between different conditions, ${ }^{\#} p<0.05$ for transfection efficiency of all conditions relative to condition " $A$ ".

the presence of interfering hydrogel materials and trypsin in the 2D cell culture media, but not by the encapsulation process since there were no statistically significant differences in transfection values between released nioplexes from degraded hydrogels and fresh nioplexes supplemented with degraded hydrogel materials (Fig. 5B and C).

Finally, gene delivery mediated by entrapped nioplexes was evaluated in hydrogels 1 (no nioplexes), 2 (0.055 $\mu \mathrm{g}$ DNA/ $\mu \mathrm{l}$ hydrogel) and $3(0.12 \mu \mathrm{g}$ DNA $/ \mu l$ hydrogel). Two main mechanisms are postulated to contribute to the gene transfer process from hydrogel scaffolds: DNA/nanoparticle release kinetics and rate of cellular infiltration. ${ }^{36}$ Nioplexes released after hydrogel degradation could transfect surrounding cells, while infiltrating cells would encounter entrapped nioplexes and become transfected leading to transgene expression inside the hydrogel area. ${ }^{37,38}$ As expected, in the present work the release kinetics of nioplexes were faster in the presence of Collagenase I ( $\mathrm{Col} \mathrm{I}$ ), with almost $100 \%$ of nioplexes being released by day in presence of Col I, compared to $\sim 80 \%$ release by day 7 in PBS alone (Fig. 6A). This progressive release of nioplexes potentially allows for sustained transgene expression, which is essential for therapeutic applicability. Regarding cellular infiltration, for cells cultured in three dimensions, the migration rate of cells through the hydrogel has previously been related to successful non-viral gene transfer. ${ }^{36}$ Therefore, we would expect that softer hydrogel scaffolds that allowed for extensive cell spreading would result in enhanced gene transfer. Interestingly, in the present work all hydrogels allowed for extensive cell spreading (Fig. 6C) and, despite its high stiffness, the 3D transfection efficiency was significantly $(p<0.05)$ higher in hydrogel 3 compared to its softer counterparts (Fig. 6B, bars). Therefore, these results suggest that increasing amounts of DNA can be used to overcome limitations of stiffer hydrogels. Moreover, cell viability was excellent in all conditions (Fig. 6B, dots), which indicated that the presence of nioplexes in the HA hydrogels was well tolerated by the cells.

\section{Conclusions}

We successfully developed a process to load concentrated nioplexes into HA hydrogels without aggregation. To our knowledge, this is the first time that niosome formulations composed
A

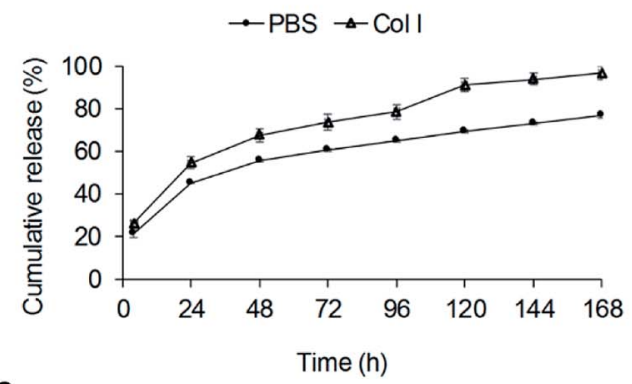

B

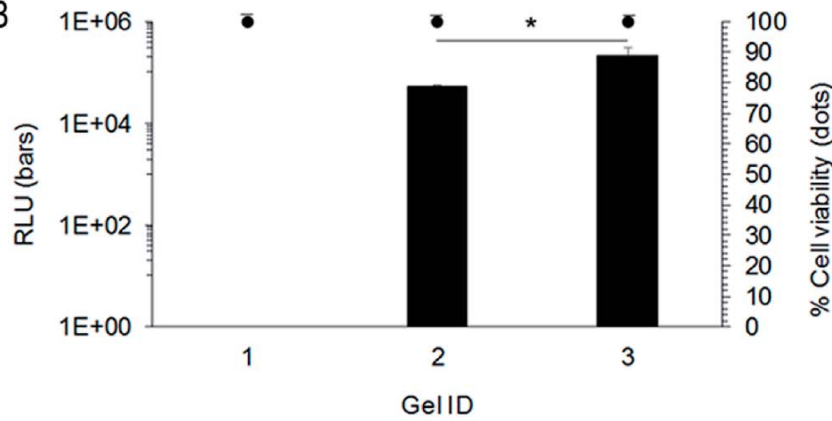

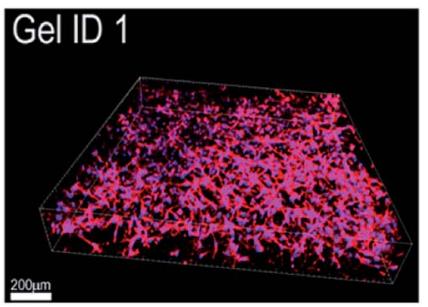
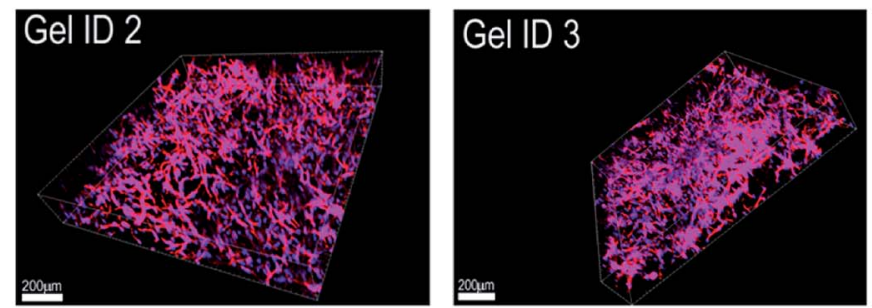

Fig. 6 (A) Nioplex release kinetics from HA hydrogels in PBS and in PBS supplemented with collagenase I (1 U ml $\left.\mathrm{l}^{-1}\right)$. (B) Transfection efficiency and cell viability of nioplex-loaded hydrogels $1-3$ at 48 h. (C) Representative images of cell spreading in hydrogels 1,2 and 3 . Blue $=$ cell nuclei (DAPI) and red = F-actin (rhodamine-phalloidin). Scale bars: $200 \mu \mathrm{m}$. ${ }^{*} p<0.05$ for transfection efficiency. 
of 2,3-di(tetradecyloxy)propan-1-amine, poloxamer 407, polysorbate 80 and chloroquine diphosphate salt are incorporated to HA hydrogels for non-viral gene delivery. In general, HA hydrogel scaffolds loaded with nioplexes presented suitable mechanical properties, little or no particle aggregation, allowed for extensive cell spreading and were able to efficiently transfect encapsulated mMSCs in 3D cultures. We believe that the knowledge gained through this in vitro model can be utilized to design novel and effective platforms for in vivo local and nonviral gene delivery applications.

\section{Conflicts of interest}

There are no conflicts to declare.

\section{Acknowledgements}

This project was supported by the Basque Country Government (Department of Education, University and Research, predoctoral grant PRE_2016_2_0302 and Consolidated Groups, IT90716) and by the Ikerbasque Foundation for Science from the Basque Country. Authors wish to thank the intellectual and technical assistance from the ICTS "NANBIOSIS", more specifically by the Drug Formulation Unit (U10) of the CIBER in Bioengineering, Biomaterials and Nanomedicine (CIBER-BBN) at the University of the Basque Country (UPV/EHU). The authors thank for technical and human support provided by SGIker of UPV/EHU and European funding (ERDF and ESF). We thank the National Institutes of Health for funding R01HL110592 (TS). I. V. B. thanks the Basque Country Government for the granted fellowship. Authors wish to thank Katrina Wilson for her technical assistance with the NMR data.

\section{References}

1 L. De Laporte and L. D. Shea, Adv. Drug Delivery Rev., 2007, 59, 292-307.

2 M. D. Krebs, O. Jeon and E. Alsberg, J. Am. Chem. Soc., 2009, 131, 9204-9206.

3 Y. Lei, S. Huang, P. Sharif-Kashani, Y. Chen, P. Kavehpour and T. Segura, Biomaterials, 2010, 31, 9106-9116.

4 L. Jin, X. Zeng, M. Liu, Y. Deng and N. He, Theranostics, 2014, 4, 240-255.

5 G. Puras, M. Mashal, J. Zárate, M. Agirre, E. Ojeda, S. Grijalvo, R. Eritja, A. Diaz-Tahoces, G. MartínezNavarrete, M. Avilés-Trigueros, E. Fernández and J. L. Pedraz, J. Controlled Release, 2014, 174, 27-36.

6 E. Ojeda, G. Puras, M. Agirre, J. Zárate, S. Grijalvo, R. Eritja, G. Martínez-Navarrete, C. Soto-Sánchez, A. Diaz-Tahoces, M. Avilés-Trigueros, E. Fernández and J. L. Pedraz, Biomaterials, 2016, 77, 267-279.

7 N. Grimaldi, F. Andrade, N. Segovia, L. Ferrer-Tasies, S. Sala, J. Veciana and N. Ventosa, Chem. Soc. Rev., 2016, 45, 65206545.

8 P. P. Karmali and A. Chaudhuri, Med. Res. Rev., 2007, 27, 696-722.
9 P. Dabkowska, D. J. Barlow, R. A. Campbell, A. V. Hughes, P. J. Quinn and M. J. Lawrence, Biomacromolecules, 2012, 13, 2391-2401.

10 F. Liu, J. Yang, L. Huang and D. Liu, Pharm. Res., 1996, 13, 1642-1646.

11 E. Ojeda, M. Agirre, I. Villate-Beitia, M. Mashal, G. Puras, J. Zárate and J. L. Pedraz, Methods Mol. Biol., 2016, 1445, 63-75.

12 J. R. Fraser, T. C. Laurent and U. B. Laurent, J. Intern. Med., 1997, 242, 27-33.

13 J. B. Leach, K. A. Bivens, C. W. Patrick and C. E. Schmidt, Biotechnol. Bioeng., 2003, 82, 578-589.

14 P. Lei, R. M. Padmashali and S. T. Andreadis, Biomaterials, 2009, 30, 3790-3799.

15 J. M. Saul, M. P. Linnes, B. D. Ratner, C. M. Giachelli and S. H. Pun, Biomaterials, 2007, 28, 4705-4716.

16 Y. G. Lei and T. Segura, Biomaterials, 2009, 30, 254-265.

17 J. A. Wieland, T. L. Houchin-Ray and L. D. Shea, J. Controlled Release, 2007, 120, 233-241.

18 S. Grijalvo, A. Alagia, G. Puras, J. Zárate, J. Mayr, J. L. Pedraz, R. Eritja and D. Díaz Díaz, J. Mater. Chem. B, 2017, 5, 77567767.

19 N. F. Truong and T. Segura, ACS Biomater. Sci. Eng., 2018, 4, 981-987.

20 N. Bhattarai and J. G. M. Zhang, Adv. Drug Delivery Rev., 2010, 62, 83-99.

21 T. Tokatlian, C. Camb and T. Segura, Biomaterials, 2014, 35, 825-835.

22 S. Taranejoo, R. Chandrasekaran, W. Cheng and K. Hourigan, Carbohydr. Polym., 2016, 153, 160-168.

23 S. Moghassemi and A. Hadjizadeh, J. Controlled Release, 2014, 185, 22-36.

24 M. Mashal, N. Attia, G. Puras, G. Martínez-Navarrete, E. Fernández and J. L. Pedraz, J. Controlled Release, 2017, 254, 55-64.

25 D. Monti, S. Burgalassi, M. S. Rossato, B. Albertini, N. Passerini, L. Rodriguez and P. Chetoni, Int. J. Pharm., 2010, 400, 32-36.

26 C. Yang, T. Hu, H. Cao, L. Zhang, P. Zhou, G. He, X. Song, A. Tong, G. Guo, F. Yang, X. Zhang, Z. Qian, X. Qi, L. Zhou and Y. Zheng, Mol. Pharm., 2015, 12, 2167-2179.

27 S. Vanesa, T. Carmen, S. Brigitte, F. Emmanuel, M. C. Helen, S. Ravi and P. McFadden, Carbon, 2011, 49, 5348-5358.

28 J. Cheng, R. Zeidan, S. Mishra, A. Liu, S. H. Pun, R. P. Kulkarni, G. S. Jensen, N. C. Bellocq and M. E. Davis, J. Med. Chem., 2006, 49, 6522-6533.

29 H. Hosseinkhani and T. Tabata, J. Nanosci. Nanotechnol., 2006, 6, 2320-2328.

30 O. Paecharoenchau, N. Niyomtham, T. Ngawhirunpat, T. Rojanarate, B. E. Yingyongnarongkul and P. Opanasopit, J. Drug Targeting, 2012, 20, 783-792.

31 L. Li, H. Song, K. Luo, B. He, Y. Nie, Y. Yang, Y. Wu and Z. Gu, Int. J. Pharm., 2011, 408, 183-1890.

32 S. Kachi, Y. Oshima, N. Esumi, M. Kachi, B. Rogers, D. J. Zack and P. A. Campochiaro, Gene Ther., 2005, 12, 843-851. 
33 E. Ojeda, G. Puras, M. Agirre, J. Zárate, S. Grijalvo, R. Pons, R. Eritja, G. Martínez-Navarrete, C. Soto-Sanchez, E. Fernández and J. L. Pedraz, Org. Biomol. Chem., 2015, 13, 1068-1081.

34 G. Caracciolo and H. Amenitsch, Eur. Biophys. J., 2012, 41, 815-829.

35 P. Moshayedi, L. R. Nih, I. L. Llorente, A. R. Berg, J. Cinkornpumin, W. E. Lowry, T. Segura and S. T. Carmichael, Biomaterials, 2016, 105, 145-155.
36 J. A. Shepard, A. Huang, A. Shikanov and L. D. Shea, J. Controlled Release, 2010, 146, 128-135.

37 Y. Lei, S. Gojgini, J. Lam and T. Segura, Biomaterials, 2011, 32, 39-47.

38 S. Gojgini, T. Tokatlian and T. Segura, Mol. Pharm., 2011, 8, 1582-1591. 